\title{
Enhanced long-range correlations of coherent waves reflected from disordered media
}

\author{
M. Nieto-Vesperinas and J. A. Sanchez-Gil \\ Instituto de Optica del Consejo Superior de Investigaciones Cientificas, Serrano 121, Madrid 28006, Spain
}

(Received 3 January 1992)

\begin{abstract}
We study the intensity angular correlation function of scalar waves, scattered on reflection from disordered media, for the case in which the sample is so small that the speckle pattern does not follow a joint Gaussian statistics, and thus the factorization approximation does not hold. An enhanced long-range correlation is predicted that exhibits two peaks due to coherent effects analogous to those producing the phenomenon of enhanced backscattering of the mean intensity as a result of time-reversal symmetry.
\end{abstract}

There has been in recent years great interest in the subject of propagation of classical waves from disordered media. $^{1-3}$ This has been mainly due to the discovery of coherent effects associated with multiple scattering, like the phenomenon of enhanced backscattering, related with the weak localization of waves, and found both in dense media ${ }^{4-9}$ and rough surfaces; ${ }^{10-13}$ and also with the prediction and observation of intensity correlations in light and other classical waves both reflected and transmitted in dense media. ${ }^{14-24}$

In this paper we address the angular correlation of the speckled intensities of coherent scalar waves reflected from a random medium under two different directions of propagation of the incident wave. We consider the case in which the sample is so small that the wave field diffusely reflected does not have a circular Gaussian joint probability density; namely, the factorization approximation is not valid. In this case, corrections to the so-called memory effect obtained under the factorization approximation have predicted the existence of long-range correlations both in reflected and transmitted waves. ${ }^{15,17,22,23}$ On the other hand, in this work we put forward the existence of a long-range angular correlation of the intensity, different from that studied in previous works, and that has the very interesting feature of being due to timereversal symmetry and having an origin analogous to the effect of enhanced backscattering in the first-order moment of the reflected intensity (namely, in the average of this quantity), as it arises from constructive interference between waves traveling paths in the random medium which are reversed of each other. In this connection, it should be remarked that Refs. 21 and 22 have considered previously some effects in the angular correlations caused by the time-reversal symmetry, but the study presented in this paper is independent of them.

We shall consider a plane wave with wave vector $\mathbf{k}_{i}$, incident on a sample of the random medium. The speckle pattern resulting from the wave reflected is considered at a generic scattering direction characterized by the wave vector $\mathbf{k}_{f}$. Subsequently, we consider another speckle pattern in the generic direction $\mathbf{k}_{f}^{\prime}$ produced on reflection of a second plane-wave incident with wave vestor $\mathbf{k}_{i}^{\prime}$. The intensity correlation function is then given by

$$
C\left(\mathbf{k}_{i}, \mathbf{k}_{f} ; \mathbf{k}_{i}^{\prime}, \mathbf{k}_{f}^{\prime}\right)=\frac{\left\langle\delta I\left(\mathbf{k}_{i}, \mathbf{k}_{f}\right) \delta I\left(\mathbf{k}_{i}^{\prime}, \mathbf{k}_{f}^{\prime}\right)\right\rangle}{\left\langle I\left(\mathbf{k}_{i}, \mathbf{k}_{f}\right)\right\rangle\left\langle I\left(\mathbf{k}_{i}^{\prime}, \mathbf{k}_{i}^{\prime}\right)\right\rangle},
$$

where $\delta I=I-\langle I\rangle$, and $\langle\cdots\rangle$ denotes ensemble average.

The numerator of Eq. (1) is obtained as follows: Let $A_{l m}$ be the complex amplitude from the initial scattering point $\mathbf{r}_{l}$ to the terminal scattering point $\mathbf{r}_{m}$, the incident and emerging wave vectors being $\mathbf{k}_{i}$ and $\mathbf{k}_{f}$, respectively. In the multiple-scattering model, one must also consider the time-reversed path from $\mathbf{r}_{m}$ to $\mathbf{r}_{l}$ whose corresponding complex amplitude is $A_{m l}$. Then the scattered field given by all these pairs of paths is

$$
\begin{aligned}
A\left(\mathbf{k}_{i}, \mathbf{k}_{f}\right)=\frac{1}{2} \sum_{l, m}\left\{A_{l m} \exp \left[i\left(\mathbf{k}_{i} \cdot \mathbf{r}_{l}-\mathbf{k}_{f} \cdot \mathbf{r}_{m}\right)\right]\right. \\
\left.+A_{m l} \exp \left[i\left(\mathbf{k}_{i} \cdot \mathbf{r}_{m}-\mathbf{k}_{f} \cdot \mathbf{r}_{l}\right)\right]\right\},
\end{aligned}
$$

and the intensity of the reflected wave is therefore

$$
\begin{aligned}
I\left(\mathbf{k}_{i}, \mathbf{k}_{f}\right)= & I_{0}+\sum_{l, m}\left|A_{l m}\right|^{2} \cos \left(\mathbf{k}_{i}+\mathbf{k}_{f}\right) \cdot\left(\mathbf{r}_{l}-\mathbf{r}_{m}\right) \\
& +F\left(\mathbf{k}_{i}, \mathbf{k}_{f}\right),
\end{aligned}
$$

where $I_{0}=\sum_{l, m}\left|A_{l m}\right|^{2}$. The second, diagonal, term of Eq. (3) leads on averaging over several realizations of the random medium to the effect of enhanced backscattering, and the third, off-diagonal, term is

$$
\begin{aligned}
F\left(\mathbf{k}_{i}, \mathbf{k}_{f}\right)=\frac{1}{4} \sum_{(l, m) \neq\left(l^{\prime}, m^{\prime}\right)} & A_{l m} A_{l^{\prime} m^{\prime}}^{*} \exp \left[i \mathbf{k}_{i} \cdot\left(\mathbf{r}_{l}-\mathbf{r}_{l^{\prime}}\right)\right] \\
& \times \exp \left[-i \mathbf{k}_{f} \cdot\left(\mathbf{r}_{m}-\mathbf{r}_{m^{\prime}}\right)\right]
\end{aligned}
$$

which represents the speckle fluctuations and is zero on averaging.

From Eq. (3) one obtains

$$
\begin{aligned}
\left\langle\delta I\left(\mathbf{k}_{i}, \mathbf{k}_{f}\right) \delta I\left(\mathbf{k}_{i}^{\prime}, \mathbf{k}_{f}^{\prime}\right)\right\rangle= & \frac{1}{2} \sum_{l, m ; j, k}\left\langle\delta I_{l m} \delta I_{j k}\right\rangle\left\{\cos \left[\left(\mathbf{k}_{i}+\mathbf{k}_{f}\right) \cdot\left(\mathbf{r}_{l}-\mathbf{r}_{m}\right)+\left(\mathbf{k}_{i}^{\prime}+\mathbf{k}_{f}^{\prime}\right) \cdot\left(\mathbf{r}_{j}-\mathbf{r}_{k}\right)\right]\right. \\
& \left.\quad+\cos \left[\left(\mathbf{k}_{i}+\mathbf{k}_{f}\right) \cdot\left(\mathbf{r}_{l}-\mathbf{r}_{m}\right)-\left(\mathbf{k}_{i}^{\prime}+\mathbf{k}_{f}^{\prime}\right) \cdot\left(\mathbf{r}_{j}-\mathbf{r}_{k}\right)\right]\right\} \\
& +\sum_{j k}\left\langle F\left(\mathbf{k}_{i}, \mathbf{k}_{f}\right) I_{j k}\right\rangle \cos \left(\mathbf{k}_{i}^{\prime}+\mathbf{k}_{f}^{\prime}\right) \cdot\left(\mathbf{r}_{j}-\mathbf{r}_{k}\right) \\
& +\sum_{l m}\left\langle F\left(\mathbf{k}_{i}^{\prime}, \mathbf{k}_{f}^{\prime}\right) I_{l m}\right\rangle \cos \left(\mathbf{k}_{i}+\mathbf{k}_{f}\right) \cdot\left(\mathbf{r}_{l}-\mathbf{r}_{m}\right)+\left\langle F\left(\mathbf{k}_{i}, \mathbf{k}_{f}\right) F\left(\mathbf{k}_{i}^{\prime}, \mathbf{k}_{f}^{\prime}\right)\right\rangle
\end{aligned}
$$


where $I_{l m}=\left|A_{l m}\right|^{2}$ and $\delta I_{l m}=I_{l m}-\left\langle I_{l m}\right\rangle$.

The diagonal elements $l=j, m=k$ or $l=k, m=j$ of the first term of Eq. (5) lead to

$$
\begin{aligned}
\sum_{l, m}\left\langle\delta I_{l m}^{2}\right\rangle\left[\cos \left[\left(\mathbf{k}_{i}+\mathbf{k}_{f}\right)+\left(\mathbf{k}_{i}^{\prime}+\mathbf{k}_{f}^{\prime}\right)\right] \cdot\left(\mathbf{r}_{l}-\mathbf{r}_{m}\right)\right. \\
\left.+\cos \left[\left(\mathbf{k}_{i}+\mathbf{k}_{f}\right)-\left(\mathbf{k}_{i}^{\prime}+\mathbf{k}_{f}^{\prime}\right)\right] \cdot\left(\mathbf{r}_{l}-\mathbf{r}_{m}\right)\right] .
\end{aligned}
$$

Equation (6) represents two peaks, centered at values

$$
\mathbf{q}_{i}+\mathbf{q}_{f}= \pm\left(\mathbf{q}_{i}^{\prime}+\mathbf{q}_{f}^{\prime}\right)
$$

where $q$ represents the transversal component of $\mathbf{k}$. An argument analogous to that used for the analysis of the enhanced backscattering line shape (cf., e.g., Ref. 7 on using a continuous approach) gives a width of these peaks of the order of $\lambda / l$, where $l$ is the transport mean free path. For fixed $\mathbf{q}_{i}^{\prime}, \mathbf{q}_{f}^{\prime}$ and $\mathbf{q}_{i}$, these two peaks represent enhanced long-range correlations (ELRC) around the two values of $\mathbf{q}_{f}$ given by Eq. (7).

Also, it is worth observing that on writing

$$
\begin{aligned}
& \left\langle I\left(\mathbf{k}_{i}, \mathbf{k}_{f}\right) I\left(\mathbf{k}_{i}^{\prime}, \mathbf{k}_{f}^{\prime}\right)\right\rangle \\
& \quad=\left\langle\left[A\left(\mathbf{k}_{i}, \mathbf{k}_{f}\right) A^{*}\left(\mathbf{k}_{i}^{\prime}, \mathbf{k}_{f}^{\prime}\right)\right]\left[A^{*}\left(\mathbf{k}_{i}, \mathbf{k}_{f}\right) A\left(\mathbf{k}_{i}^{\prime}, \mathbf{k}_{f}^{\prime}\right)\right]\right\rangle,
\end{aligned}
$$

one can see that the term described by Eq. (6) arises from interference of terms of the form

$$
\left|A_{l m}\right|^{2} \exp \left[i\left(\mathbf{k}_{i}-\mathbf{k}_{i}^{\prime}\right) \cdot \mathbf{r}_{l}\right] \exp \left[-i\left(\mathbf{k}_{f}-\mathbf{k}_{f}^{\prime}\right) \cdot \mathbf{r}_{m}\right]
$$

and their time-reversed counterparts, and also of terms

$$
\left|A_{l m}\right|^{2} \exp \left[i\left(\mathbf{k}_{i}+\mathbf{k}_{f}^{\prime}\right) \cdot \mathbf{r}_{l}\right] \exp \left[-i\left(\mathbf{k}_{i}^{\prime}+\mathbf{k}_{f}\right) \cdot \mathbf{r}_{m}\right]
$$

and their time reversed. Observe also that the usual factorization approximation does not allow for this interference effect and, hence, leads to the memory effect (ME) only. In this sense, the ME is a "specular" phenomenon in $\Delta \mathbf{k}_{i}=\mathbf{k}_{i}-\mathbf{k}_{i}^{\prime}$ and $\Delta \mathbf{k}_{f}=\mathbf{k}_{f}-\mathbf{k}_{f}^{\prime}$, and so is the timereversed $M E$ in $\mathbf{k}_{i}+\mathbf{k}_{f}^{\prime}$ and $\mathbf{k}_{i}^{\prime}+\mathbf{k}_{f}$.

Concerning the other terms of Eq. (5), the averages in the second and third terms are zero, whereas the fourth term is zero for $j=l, k=m, j^{\prime}=l^{\prime}, k^{\prime}=m^{\prime}$ or $j=m$, $j^{\prime}=m^{\prime}, k=l, k^{\prime}=l^{\prime}$, and leads to the ME (terms with $j=l^{\prime}, k=m^{\prime}, j^{\prime}=l, k^{\prime}=m$ ) and its time reversal (terms with $\left.j=m^{\prime}, k=l^{\prime}, j^{\prime}=m, k^{\prime}=l\right)$. The other long-range correlations, addressed in Ref. 17, should be included in the other surviving elements of Eq. (5).

The ratio of the peak values of the ELRC and the ME is $\Sigma_{l m}\left\langle\delta I_{l m}^{2}\right\rangle /\left[\Sigma_{l m}\left\langle I_{l m}\right\rangle\right]^{2}$. The ELRC effect should be observed in speckle patterns corresponding to a scattered field on reflection that does not have a Gaussian joint probability density for its random values at two pairs of arguments $\left(\mathbf{k}_{i}, \mathbf{k}_{f}\right)$ and $\left(\mathbf{k}_{i}^{\prime}, \mathbf{k}_{f}^{\prime}\right)$, and, hence, for which the factorization approximation does not hold. As said in the Introduction, such speckle patterns are produced by waves backscattered from samples whose linear dimension is not much larger than $l$. For instance, the ELRC are present in the experiments from small samples studied by the authors of Ref. 9 [cf., the tails in the correlations of the full lines of Fig. 3 of Ref. 9, which correspond to $C\left(\mathbf{k}_{i}, \mathbf{k}_{f} ; \mathbf{k}_{i}^{\prime}, \mathbf{k}_{f}^{\prime}\right)$ for $\mathbf{k}_{i}=\mathbf{k}_{i}^{\prime}=-\mathbf{k}_{f}^{\prime}$ and varying $\mathbf{k}_{f}$; ;1- though it seems to us that these curves were not analyzed there from the point of view taken in our work.

It is well known, since the works of Refs. 11-13 and 25 , that random rough surfaces present some scattering properties analogous to dense media; in particular, they produce coherent effects in multiple scattering, like enhanced backscattering. It is to be expected, therefore, that the coherent phenomenon predicted above should also be present both in random rough surfaces and dense media.

In order to assess the above predictions we have computed the correlation Eq. (1) in the numerical simulation of the scattering of electromagnetic waves, $s$ or $p$ polarized from a one-dimensional random rough surface, of profile $z=D(x)$, separating the vacuum from a perfect conductor (the surface thus being constant in the $y$ direction). The plane of incidence is $O X Z$. The lack of two dimensions for the surface is made for the sake of saving computation time and is not relevant to the purpose of this work since it only prevents studying crosspolarization effects under the above choice of plane of incidence and polarization of the incident wave. Also, the assumption of a perfect conductor simplifies the calculations over those concerning a penetrable medium without introducing other important restrictions for our purpose. $^{26}$

According to the Monte Carlo procedure reported in Refs. 12 and 13, we generate random profiles with Gaussian statistics, zero mean, rms height $\sigma=1.9 \lambda$, a Gaussian correlation function of the random heights, and a correlation length $T=3.16 \lambda$. Each realization of the illuminated surface is sampled with 300 sampling points and has a length $L \approx 30 \lambda$. This involves 10 sampling points per wavelength, which is accurate enough. Averages are made over 4000 realizations. Two Gaussian beams (chosen instead of plane waves in order to get smoother results), of width $W=L \cos \theta_{0} / 4$, propagating in the directions $\mathbf{k}_{i}$ and $\mathbf{k}_{i}^{\prime}$, are incident at an angle $\theta_{0}$ and $\theta_{0}^{\prime}$ with the $O Z$ axis, respectively; namely, with the surface mean plane normal. Let $\theta$ and $\theta^{\prime}$ represent the angles of $\mathbf{k}_{f}$ and $\mathbf{k}_{f}^{\prime}$ with the $O Z$ axis, respectively.

Figure 1 shows the function $C(\theta)$, Eq. (1), for $\mathbf{k}_{i}, \mathbf{k}_{i}^{\prime}$ and $\mathbf{k}_{f}^{\prime}$ fixed at $\theta_{0}=0^{\circ}, \theta_{0}^{\prime}=\theta^{\prime}=5^{\circ}$. The central peak at $\theta_{0}=0^{\circ}$ corresponds to the memory effect and has a width

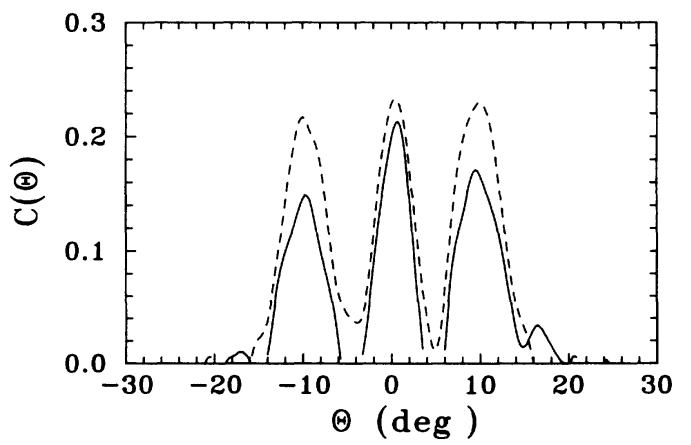

FIG. 1. Angular correlation vs $\theta$, with $\theta_{0}=0^{\circ}, \theta_{0}^{\prime}=\theta^{\prime}=5^{\circ}$. $T=3.16 \lambda$ and $\sigma=1.9 \lambda$. Dashed line: $s$-polarization. Solid line: $p$ polarization. 


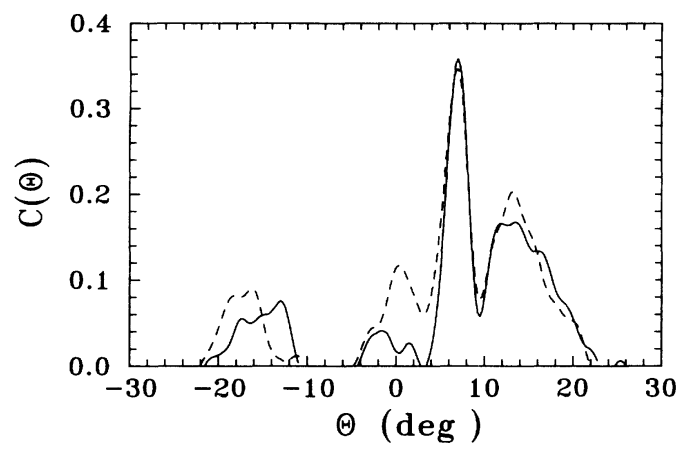

FIG. 2. Same as Fig. 1 for $\theta_{0}=2^{\circ}, \theta_{0}^{\prime}=5^{\circ}, \theta^{\prime}=9^{\circ}$.

of the order of $\cos \theta_{0} \lambda / W$. On the other hand, the two additional peaks, predicted by Eq. (6), are also present at $\theta \approx \pm 10^{\circ}$; they have a width $\lambda / T$ approximately, as $l$ is of the order of $T$ in this case (cf., Ref. 26). The strength of these peaks decreases as both $\Delta \mathbf{q}_{i}$ and $2\left[\Delta \mathbf{q}_{i}-\left(\mathbf{q}_{i}^{\prime}+\mathbf{q}_{f}^{\prime}\right)\right]$ increase. Figure 2 shows another instance for which the fourth angles $\theta_{0}, \theta_{0}^{\prime}, \theta^{\prime}$, and $\theta$ are different from each other. In this case the peak corresponding to the memory effect is at $\theta \approx 6^{\circ}\left(\theta_{0}=2^{\circ}, \theta_{0}^{\prime}=5^{\circ}, \theta^{\prime}=9^{\circ}\right)$, and those corresponding to the ELRC of Eq. (6) now appear at $\theta \approx 12^{\circ}$ and $-16^{\circ}$.

In order to analyze the multiple-scattering origin of the ELRC peaks, we have solved the Helmholtz-Kirchhoff surface-scattering integral equation by an iterative method, similar to the Born series, that accounts for the successive hits of the wave with the surface. In calculations for the first statistical moment of the intensity ${ }^{13,27,28}$ in the regime of surface parameters considered here, the sum of first- (FO) and second-order (SO) fields is usually almost sufficient to describe the scattering process. In our calculation, the result so obtained is not quantitatively identical to the exact one of Figs. 1 and 2 (this being due to a well-known lack of accuracy of the iterative method in many instances, which often requires one to add other corrections ${ }^{28}$ ); but this rather heuristic procedure still gives an illustrative estimation of the contribution of the second hit of the wave at the surface. Figure 3 shows the result for the same situation as for Fig. 1 .

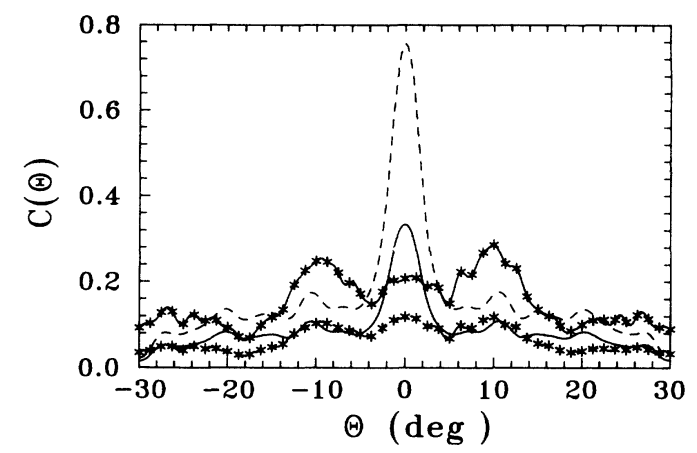

FIG. 3. Iterative computation of the angular correlation vs $\theta$, with $\theta_{0}=0^{\circ}, \theta_{0}^{\prime}=\theta^{\prime}=5^{\circ} . T=3.16 \lambda$ and $\sigma=1.9 \lambda$. Dashed line: $s$ polarization. Solid line: $p$ polarization. Without asterisks: FO contribution (single scattering). With asterisks: SO contribution (purely double scattering).

Here the part of the function $C(\theta)$ obtained with the FO field (Kirchhoff approximation, i.e., single scattering) and that obtained with the SO field (double scattering) are shown. Whereas the FO result only shows the ME peak at $\theta_{0}=0^{\circ}$, the SO contribution to the correlation exhibits a much weaker lobe corresponding to the remaining $\mathrm{ME}$ and the two remarkable lobes of the ELRC at $\theta \approx \pm 10^{\circ}$. Of course, although not shown here, we have also checked that a computation of Eq. (1) on the basis of the factorization approximation yields only the peaks of both the $\mathrm{ME}$ and the time-reversed ME, as expected.

We believe that the coherent effects, like the one addressed in this paper and the phenomenon of enhanced backscattering, are special cases for the lower statistical moments of the reflected intensity of a general class of coherent phenomena present in the moments of arbitrary order of non-Gaussian speckle patterns. Experiments should establish the limits of observation of these effects.

This work was supported by the CICYT under Grant No. PB0278. J.A.S.-G. acknowledges a grant from the MEC. Discussions with J. C. Dainty and A. A. Maradudin, supported by a NATO travel grant, are also acknowledged.
${ }^{1}$ Scattering and Localization of Classical Waves in Random Media, edited by P. Sheng (World Scientific, Singapore, 1990).

${ }^{2}$ Analogies in Optics and Micro Electronics, edited by W. Van Haeringen and D. Lestra (Kluwer Academic, The Netherlands, 1990).

${ }^{3}$ Scattering in Volumes and Surfaces, edited by M. NietoVesperinas and J. C. Dainty (North-Holland, Amsterdam, 1990).

${ }^{4}$ Y. Kuga and A. Ishimaru, J. Opt. Soc. Am. A 1, 831 (1984); L. Tsang and A. Ishimaru, ibid. 1, 836 (1984).

${ }^{5}$ H. P. Van Alvada and A. Lagendijk, Phys. Rev. Lett. 55, 2692 (1985).

${ }^{6}$ P. E. Wolf and G. Maret, Phys. Rev. Lett. 55, 2696 (1985).

${ }^{7}$ E. Akkermans, P. E. Wolf, and R. Maynard, Phys. Rev. Lett.
56, 1471 (1986); E. Akkermans, P. E. Wolf, R. Maynard, and G. Maret, J. Phys. (Paris) 49, 77 (1988).

${ }^{8}$ M. Kaveh, M. Rosenbluth, M. Edrei, and I. Freund, Phys. Rev. Lett. 57, 2049 (1986).

${ }^{9}$ S. Etemand, R. Thompson, and M. J. Andrejco, Phys. Rev. Lett. 57, 575 (1986).

${ }^{10}$ V. Celli, A. A. Maradudin, A. M. Marvin, and A. R. McGurn, J. Opt. Soc. Am. 2, 2225 (1985).

${ }^{11}$ K. A. O'Donnell and E. R. Mendez, J. Opt. Soc. Am. A 4, 1194 (1987).

${ }^{12}$ M. Nieto-Vesperinas and J. M. Soto-Crespo, Opt. Lett. 12, 979 (1987); J. M. Soto-Crespo and M. Nieto-Vesperians, J. Opt. Soc. Am. A 6, 367 (1989).

${ }^{13}$ A. A. Maradudin, E. R. Mendez, and T. Michel, Opt. Lett. 
14, 151 (1989); A. A. Maradudin, T. Michel, A. R. McGurn, and E. R. Mendez, Ann. Phys. (NY) 203, 255 (1990).

${ }^{14}$ B. Shapiro, Phys. Rev. Lett. 57, 2168 (1986).

${ }^{15}$ M. Stephen and G. Cwilich, Phys. Rev. Lett. 59, 285 (1987).

${ }^{16}$ A. Z. Genack, Phys. Rev. Lett. 58, 2043 (1987).

${ }^{17}$ S. Feng, C. Kane, P. A. Lee, and D. Stone, Phys. Rev. Lett. 61, 834 (1988).

${ }^{18}$ I. Freund, M. Rosenbluth, and S. Feng, Phys. Rev. Lett. 61, 2328 (1988).

${ }^{19}$ R. Berkovits, M. Kaveh, and S. Feng, Phys. Rev. B 40, 737 (1989).

${ }^{20}$ N. Garcia and A. Z. Genack, Phys. Rev. Lett. 63, 1678 (1989).

${ }^{21}$ R. Berkovits and M. Kaveh, Phys. Rev. B 41, 2635 (1990).
${ }^{22}$ R. Berkovits, Phys. Rev. B 42, 10750 (1990).

${ }^{23}$ M. P. Van Albada, J. F. de Boer, and A. Lagendijk, Phys. Rev. Lett. 64, 2787 (1990).

${ }^{24}$ D. Eliyahu, R. Berkovits, and M. Kaveh, Phys. Rev. B 43, 13501 (1991).

${ }^{25}$ M. J. Kim, J. C. Dainty, A. T. Friberg, and A. J. Sant, J. Opt. Soc. Am. A 7, 569 (1990).

${ }^{26}$ A. A. Maradudin, E. R. Mendez, and T. Michel, Scattering in Volumes and Surfaces (Ref. 3), p. 157.

${ }^{27}$ A. Ishimaru and J. S. Chen, J. Acoust. Soc. Am. 88, 1877 (1990).

${ }^{28}$ E. I. Thorsos and D. R. Jackson, Waves Random Media 1, S165 (1991). 